THE ASTROPHYSICAL JOURNAL, 321:L29-L33, 1987 October 1

(c) 1987. The American Astronomical Society. All rights reserved. Printed in U.S.A.

\title{
A CORRELATION BETWEEN THE RADIO AND OPTICAL MORPHOLOGIES OF DISTANT 3CR RADIO GALAXIES ${ }^{1}$
}

\author{
Patrick J. McCarthy, Wil van Breugel, and Hyron SpinRad ${ }^{2,3}$ \\ Astronomy Department, University of California, Berkeley \\ AND \\ S. DJORGovski ${ }^{2,3,4}$ \\ Harvard-Smithsonian Center for Astrophysics \\ Received 1987 June 5; accepted 1987 July 20
}

\begin{abstract}
We report the discovery of a strong correlation between the radio and optical morphologies of distant $(z \geq 0.6)$, powerful radio galaxies. The isophotal axes of highly elongated distant 3CR galaxies, measured both in the light of stellar continua and extranuclear emission lines, tend to align with the radio source axes. This correlation suggests that there is a profound relation between these extremely powerful radio sources and their host galaxies. We propose that the most natural explanation of the effect is that the radio jets and/or backflows from the radio lobes interact with the interstellar media of the gas-rich galaxies associated with 3CR sources and stimulate large-scale star formation. This proposed mechanism can provide a physical explanation for the high star formation and evolution rates of 3CR galaxies at large look-back times found in earlier photometric studies. Thus, the formation and evolution of powerful radio galaxies and radio-quiet giant ellipticals may differ substantially.
\end{abstract}

Subject headings: cosmology — galaxies: formation — galaxies: jets — galaxies: structure radio sources: galaxies — radio sources: general

\section{INTRODUCTION}

During the past several years much new information concerning the optical properties of radio galaxies has become available. Many results pertain almost exclusively to optically bright, nearby $(z<0.05)$, and hence primarily low power $\left(P_{408 \mathrm{MHz}} \approx 10^{23.5}-10^{25.5} \mathrm{~W} \mathrm{~Hz}^{-1}\right)$ sources. Morphological studies have shown that radio galaxies tend to be situated in elliptical galaxies that are on average rounder and redder than their radio quite counterparts (e.g., Cordey 1986 and references therein). Early reports of alignments between the galaxy major axis and the radio source axis by Mackay (1971) were called into question by Bridle and Brandie (1973). More recent investigations have shown that nearby radio galaxies with relatively bright radio cores tend to have their radio axes along the minor axes of their parent galaxies (Kapahi and Saikia 1982, and references therein).

Heckman et al. (1986) and Hutchings (1987) have found that a large fraction of radio galaxies with redshifts up to 0.3 , and radio powers $P_{408 \mathrm{MHz}}>10^{25.5} \mathrm{~W} \mathrm{~Hz}^{-1}$ have peculiar morphologies suggestive of galaxy collisions or merging. Many of these have associated optical emission-line regions

\footnotetext{
${ }^{1}$ Based in part on data obtained at Lick Observatory, University of California.

${ }^{2}$ Visiting Astronomer, Kitt Peak National Observatory, NOAO, operated by AURA, Inc., under contract with NSF.

${ }^{3}$ Visiting Astronomer, Canada-France-Hawaii Telescope, operated by the NRC (Canada), CNRS (France), and the University of Hawaii.

${ }^{4}$ Society of Fellows, Harvard University.
}

(van Breugel 1986), which are often, but not always (cf. Baum 1986) found along the radio source axes. Star formation, presumably induced by the passage of the jets through regions of dense gas, has been found along the radio jet trajectories in Centaurus A (Graham and Price 1981) and Minkowski's Object (van Breugel et al. 1985; Brodie, Bowyer, and McCarthy 1985).

Considerable progress has recently been made in observations of very distant $(z>0.5)$, powerful $\left(P_{408 \mathrm{MHz}}>\right.$ $10^{25.5} \mathrm{~W} \mathrm{~Hz}^{-1}$ ) 3CR radio galaxies (Spinrad and Djorgovski $1984 a, b$; Spinrad et al. 1985). Photometry of these distant galaxies shows evidence for evolutionary effects in their stellar populations (Lilly and Longair 1984; Spinrad 1986; Djorgovski 1987). High star formation rates (in excess of $100 M_{\odot}$ $\mathrm{yr}^{-1}$ ) are indicated in some cases, and it is likely that we are seeing these objects as they undergo spectacular starbursts, the origin of which is yet to be determined.

In this Letter we report results of our radio/optical investigations of a nearly complete sample of very distant radio galaxies. We find a surprisingly strong alignment between the radio source axes and the optical major axes of the parent galaxies. This correlation indicates that a simple merger hypothesis is insufficient in explaining the properties of highredshift radio galaxies, and suggests a fundamental relationship between young active galaxies and their radio sources.

\section{OBSERVATIONS AND ANALYSIS}

The data, deep $B, V$, and $R$ CCD images, were obtained primarily with the Kitt Peak National Observatory (KPNO) 
Mayall $4 \mathrm{~m}$ telescope and with the Canada-France-Hawaii telescope. The details of the observing procedures and reductions will be given by S. Djorgovski, H. Spinrad, and $M$. Dickinson (in preparation). Additional CCD images were obtained by us with the Lick Observatory Shane $3 \mathrm{~m}$ telescope.

To define a large, yet nearly complete, sample we have restricted ourselves to galaxies in the redshift range from 0.6 to the most distant $3 \mathrm{CR}$ radio galaxy currently known, $3 \mathrm{C}$ 326.1 ( $z=1.825$; McCarthy et al. 1987). High-quality direct images are available for $80 \%$ of this sample. Of the $583 \mathrm{CR}$ galaxies with $z>0.6,24$ have isophotes that are sufficiently elongated to allow a determination of the position angles of their major axes. The position angle and size of each galaxy were determined at the $6 \%$ sky brightness isophote from contour plots of the images. Repeated measurements indicate that the position angles are accurate to about $5^{\circ}-15^{\circ}$. Typical sizes at $6 \%$ sky for $z \approx 1$ are $2^{\prime \prime} \times 5^{\prime \prime}$, corresponding to $\sim 20 \times 50 \mathrm{kpc}$ for $H_{0}=50, q_{0}=0$.

For objects with redshifts between 0.6 and 0.9 the $R$ band images will contain contributions from [O II] $\lambda 3727$. Spinrad (1986) shows that with few exceptions 3CR galaxies in this redshift range have $W_{\lambda}(3727) \leq 350 \AA$, and thus the [O II] emission may make an important contribution to the broadband images only in the strongest lined galaxies. We have used $V$ images for the galaxies in this redshift range whenever possible. For the more distant galaxies, at $z>1$, no strong emission lines fall within either the $B, V$, or $R$ filters.

We have imaged 18 of the galaxies in the sample with interference filters centered on [O II] $\lambda 3727$. The extended [O II] regions are often complex; thus their position angles are less well defined and have uncertainties of $\sim 20^{\circ}-25^{\circ}$. An extensive study of the extended [O II] emission, optical, and radio continua in $3 \mathrm{C} 368(z=1.132)$ has recently been published by Djorgovski et al. (1987). A detailed discussion of our larger [O II] imaging program will be presented elsewhere.

For each galaxy in the sample we also determined the size and position angle of its large-scale radio emission using maps published in the literature, primarily obtained with the Cambridge $5 \mathrm{~km}$ telescope (Jenkins, Pooley, and Riley 1977). At the large redshifts of our sample all of the radio galaxies are powerful and have edge-brightened (Fanaroff-Riley Class II) morphologies. Because of their simple morphologies, the radio source position angles are usually better defined, and more accurate $\left(\sim 2^{\circ}-3^{\circ}\right)$, than the isophotal position angles of the parent galaxies. The measured optical and radio position angles of the program galaxies are listed in Table 1.

\section{RESULTS AND DISCUSSION}

One of the most intriguing results of the broad-band imaging is that a large fraction of the distant radio galaxies have highly elongated or multimodal shapes (Spinrad and Djorgovski 1984c; Lilly and Longair 1984; Djorgovski 1987; Spinrad 1987). For $z>1$, we find that $50 \%$ of our 3CR sample galaxies have axial ratios greater than $\sim 2$ at levels greater than $30 \%$ of their peak surface brightness. This is quite significant, because the effects of seeing and cosmological surface brightness dimming act to make distant galaxies appear to be rounder and more nucleated than when viewed at low redshifts. Two examples are shown in Figures 1 and 2 (Plates L3 and L4) (3C 324, $z=1.206$; 3C 266, $z=1.275$ ). In the redshift range $0.6-1$, only $\sim 30 \%$ of the galaxies are elongated, and their ellipticities are significantly less than for galaxies with $z>1$. This morphology should not be confused with the low surface brightness features (tidal tails, shells, etc.) reported by Heckman et al. (1986) for low-redshift powerful radio galaxies; the peculiarities that we report here are at far higher surface brightness levels and involve most of the galaxian light.

In Figure $3 a$ we show a histogram of the absolute values of the differences in optical and radio position angles for 3CR galaxies with $z>0.6$ that have both optical and radio position angles determined. In Figure $3 b$ we present a similar histogram for the extended emission-line regions that we have imaged in [O II] and Ly $\alpha$ (3C 326.1).

The correlation between the optical and radio position angles is quite striking. For $z>1.0,11$ of the 12 asymmetric $3 \mathrm{CR}$ galaxies are aligned within $25^{\circ}$ of the radio source axis. Extending the sample to redshifts of 0.6 and greater adds 15 more nonround galaxies, 12 of which are aligned within $25^{\circ}$ of the radio source axis. We note that in nearly all cases the radio sources are larger than their associated galaxies.

Even gross measurement errors are unlikely to conspire to produce such an alignment correlation from a random distribution. The $\chi^{2}$ test rules out the null hypothesis (viz., that the optical and radio position angles are uncorrelated) at a confidence level greater than $99.5 \%$, and the Kolmogorov-Smirnov test rejects the null hypothesis at greater than the $99.9 \%$ level. We note that projection effects would tend to diminish the effect, and that the correlation in three dimensions must be even stronger.

A similar alignment between radio and optical (broad-band) morphologies has recently been found independently by Chambers et al. (1987), in a different radio sample selected for high-redshift parent galaxies with very steep radio spectra. The occurrence of the alignment in both samples reinforces its significance.

In the following discussion we will consider a number of possible explanations for the alignments that we observe.

The large sizes of the extended radio source structures (compared to the optical sizes), the smooth velocity fields, and the absence of double radio cores all argue against gravitational lensing playing an important role in determining the morphologies, as has been suggested by Le Fevre et al. (1987). A scenario in which beamed radiation from an obscured active nucleus preferentially ionizes gas along the axis of a thick torus is also unlikely since the extended continuum that we see is dominated by starlight rather than emission lines or scattered nuclear light.

The highly elongated and clumpy structures of the distant radio galaxies have been interpreted in some cases as being due to violent, strongly dissipative mergers of gas-rich galaxies which can both stimulate starbursts, and feed the radio sources (Djorgovski et al. 1987; Djorgovski 1987). The observed morphologies are qualitatively similar to the types of gravitational structures seen in the Frenk et al. (1987) model simulations, which show strong merging activity and rapid dynamical evolution of galaxy and galaxy group-like structures at redshifts near unity. From the point of view of a 


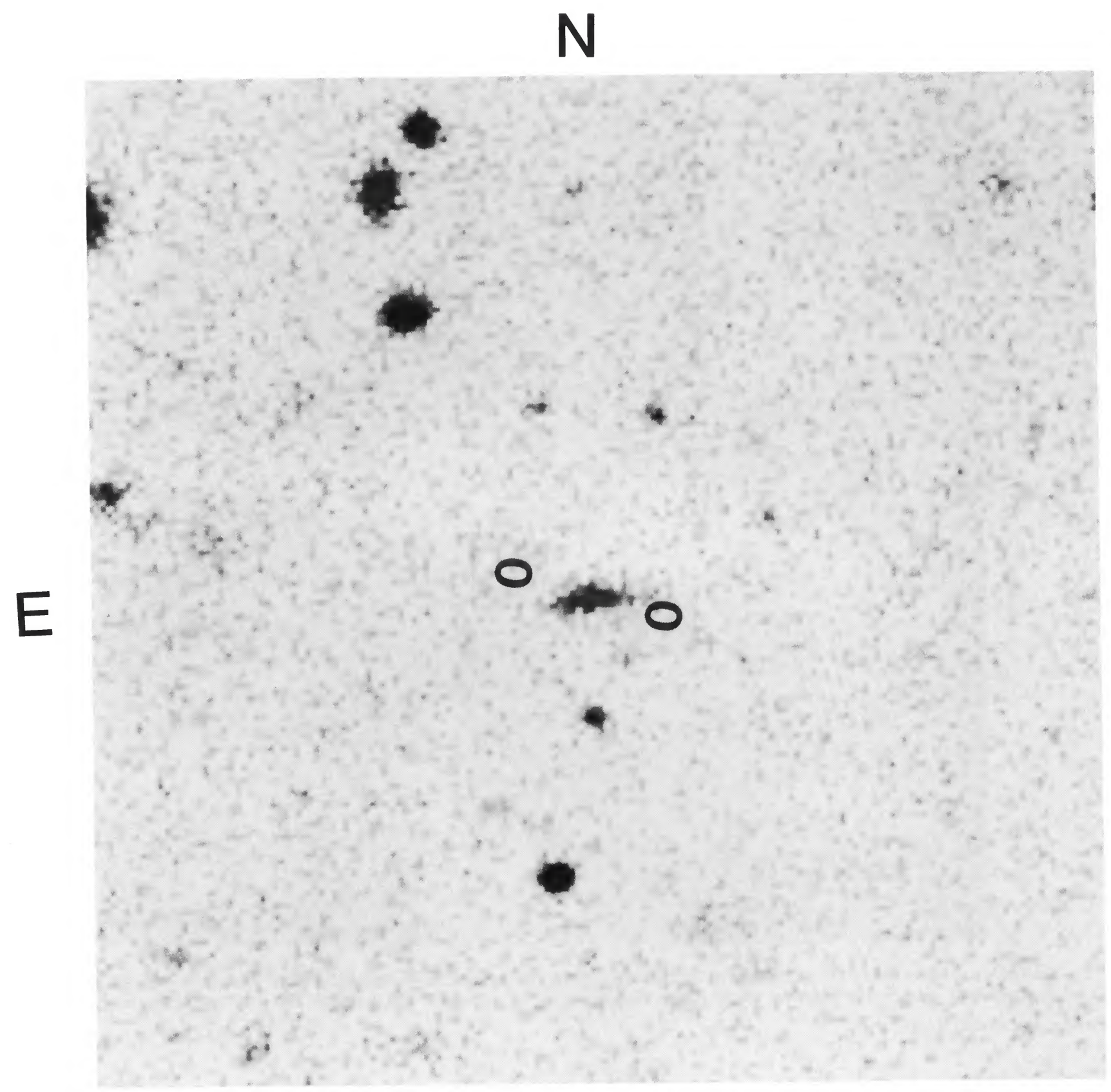

FIG. 1. - A broad-band $R$ CCD image of 3C $324(z=1.206)$. The positions of the radio hot spots are marked. This object shows the extreme elongation typical of the distant 3CR galaxies. The positional alignment with the radio source is $\sim 20^{\circ}$, also typical of the sources in the sample. The total size of the image is $49^{\prime \prime} \times 49^{\prime \prime}$.

MCCARTHY et al. (see 321, L30) 


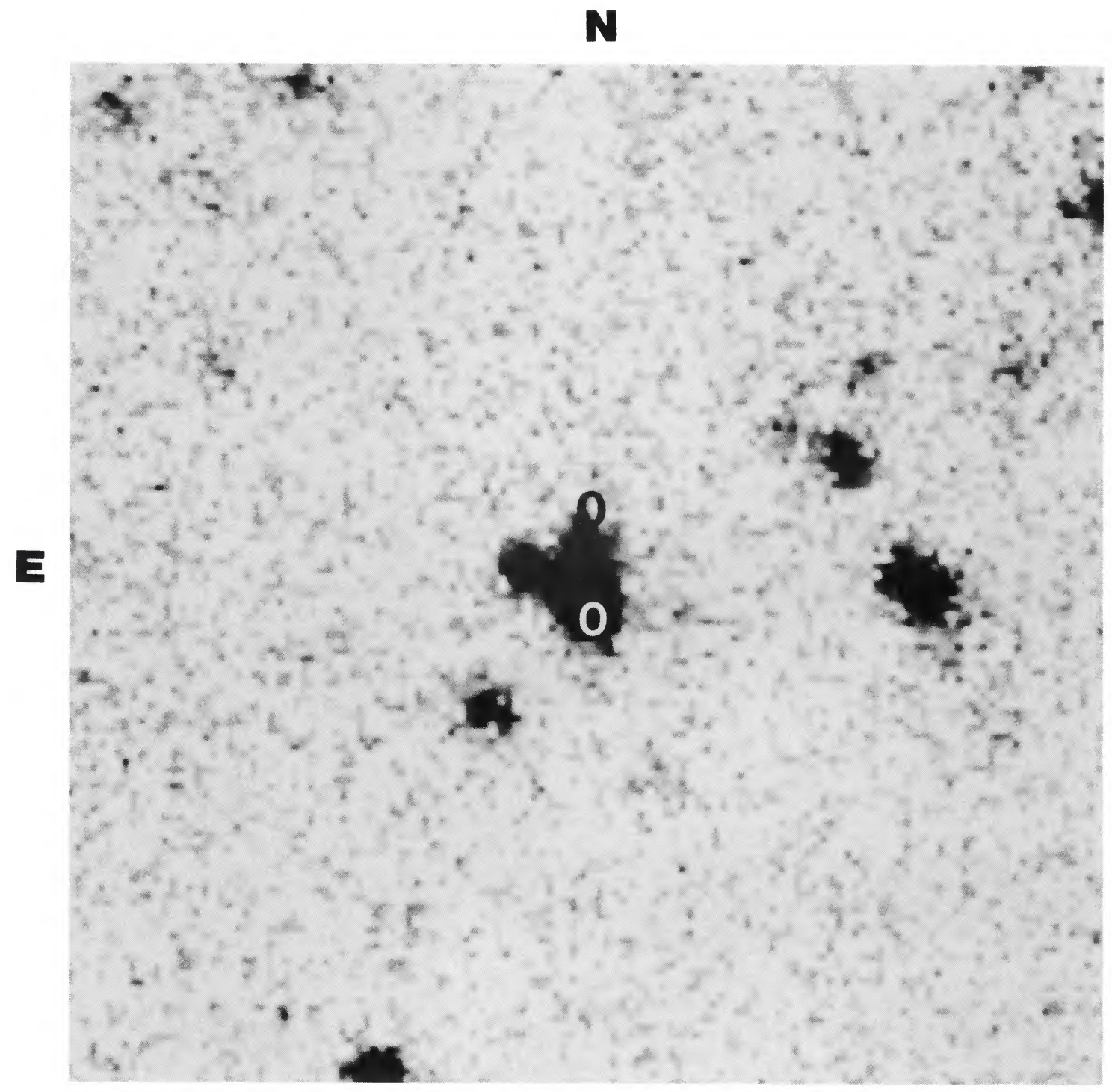

FIG. 2. - Sum of broad-band $R$ and $V$ CCD images of $3 C 266(z=1.275)$. The positions of the radio hot spots, marked by ovals, show that the radio source is aligned with and is nearly the same size as the galaxy. The object to the east of 3C 266 is stellar. The total size of the image is 38" $\times 38^{\prime \prime}$. MCCARTHY et al. (see 321, L30) 
TABLE 1

Position Angle Measurements for Program Galaxies

\begin{tabular}{|c|c|c|c|c|c|c|c|}
\hline \multirow{2}{*}{\multicolumn{2}{|c|}{ GALAXY }} & \multirow[b]{3}{*}{$\begin{array}{c}z \\
(3)\end{array}$} & \multicolumn{3}{|c|}{ PA } & \multicolumn{2}{|c|}{$|\Delta \mathrm{PA}|$} \\
\hline & & & \multirow[b]{2}{*}{$\begin{array}{c}\text { Radio } \\
\text { (4) }\end{array}$} & \multirow{2}{*}{$\begin{array}{l}\text { Optical } \\
\text { Continuum } \\
(5)\end{array}$} & \multirow{2}{*}{$\begin{array}{c}\text { Emission } \\
\text { Gas } \\
(6)\end{array}$} & \multirow{2}{*}{$\begin{array}{l}\text { Radio-Optical } \\
\text { Continuum } \\
\text { (7) }\end{array}$} & \multirow{2}{*}{$\begin{array}{c}\text { Radio-Emission } \\
\text { Gas } \\
\text { (8) }\end{array}$} \\
\hline $\begin{array}{l}\text { IAU } \\
(1)\end{array}$ & $\begin{array}{c}3 C R \\
\text { (2) }\end{array}$ & & & & & & \\
\hline $1553+202$ & 326.1 & 1.825 & 94 & 108 & 108 & 14 & 14 \\
\hline $0806+426$ & $194 \ldots$ & 1.779 & 151 & 172 & $\ldots$ & 21 & $\ldots$ \\
\hline $0031+391$ & 13. & 1.351 & 145 & 160 & $\ldots$ & 15 & $\ldots$ \\
\hline $0939+140$ & $225 \mathrm{~A}$ & 1.565 & 15 & 30 & $\ldots$ & 15 & $\ldots$ \\
\hline $2145+151$ & $437 \ldots$ & 1.480 & 160 & 122 & 120 & 38 & 40 \\
\hline $1143+500$ & $266 \ldots$ & 1.275 & 177 & 7 & $\ldots$ & 10 & $\ldots$ \\
\hline $1547+215$ & $324 \ldots$ & 1.206 & 71 & 90 & 90 & 19 & $\cdot 19$ \\
\hline $0855+280$ & $210 \ldots$ & 1.169 & 167 & 168 & & 1 & $\ldots$ \\
\hline $1147+130$ & $267 \ldots$ & 1.140 & 79 & 60 & 68 & 19 & 11 \\
\hline $1447+771$ & 305.1 & 1.132 & 11 & -10 & $\ldots$ & 21 & $\ldots$ \\
\hline $1802+110$ & $368 \ldots$ & 1.132 & 18 & 20 & 20 & 2 & 2 \\
\hline $1723+510$ & $356 \ldots$ & 1.079 & 162 & 160 & 147 & 2 & 15 \\
\hline $1256+476$ & $280 \ldots$ & 0.997 & 91 & 90 & 90 & 1 & 1 \\
\hline $0733+705$ & $184 \ldots$ & 0.994 & 106 & 83 & $\ldots$ & 23 & $\ldots$ \\
\hline $1343+500$ & $289 \ldots$ & 0.967 & 108 & 152 & $\ldots$ & 44 & $\ldots$ \\
\hline $0711+146$ & 175.1 & 0.920 & 72 & 90 & $\ldots$ & 18 & $\ldots$ \\
\hline $0013+790$ & 6.1 & 0.840 & 26 & 32 & $\ldots$ & 6 & $\ldots$ \\
\hline $0152+435$ & $54 \ldots$ & 0.827 & 25 & 20 & & 5 & $\ldots$ \\
\hline $0941+100$ & $226 \ldots$ & 0.818 & 144 & 146 & 129: & 2 & 17 \\
\hline $1142+318$ & $265 \ldots$ & 0.811 & 106 & $\mathrm{R}$ & 116 & .. & 10 \\
\hline $1709+460$ & 352. & 0.806 & 164 & 155 & 159 & 9 & 5 \\
\hline $1627+234$ & $340 \ldots$ & 0.775 & 88 & 90: & 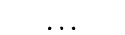 & 2: & \\
\hline $1251+159$ & 277.2 & 0.766 & 61 & 60 & 60 & 1 & 1 \\
\hline $1637+626$ & 343.1 & 0.750 & 97 & 101 & $\ldots$ & 4 & $\ldots$ \\
\hline $1056+432$ & $247 .$. & 0.749 & 69 & $55:$ & 55 & 14 & 14 \\
\hline $2203+292$ & 441 . & 0.707 & 149 & 164 & 165 & 15 & 16 \\
\hline $1627+444$ & $337 .$. & 0.635 & 103 & $\mathrm{R}$ & 118 & & 15 \\
\hline $0647+452$ & 169.1 & 0.633 & 137 & 167 & 167 & 30 & 30 \\
\hline $0926+793$ & 220.1 & 0.620 & 79 & 162: & 55: & 83: & 24: \\
\hline
\end{tabular}

NoTES. - Radio data are inadequate for 3C 256 and $3 \mathrm{C} 124$. The following sources and those marked $\mathrm{R}$ in col. (4) are round in the optical continuum: 3C 22, 36, 44, 55, 65, 68.2, 114, 208.1, 239, 241, 297, 469.1, and 470. We have no modern optical images for $3 \mathrm{C} 41,217,220.3,252,268.1$, and 292 . The following sources are too close to bright stars to be imaged effectively: 3C 230, 272, and 294. The following sources are new identifications, superseding those listed by Spinrad et al. 1985: 3C 194, 225A, 326.1, and 437. The redshifts listed in col. (3) are taken from Spinrad et al. 1985 or Spinrad et al. in preparation, except for 3C 194 which is from Djorgovski et al. in preparation.

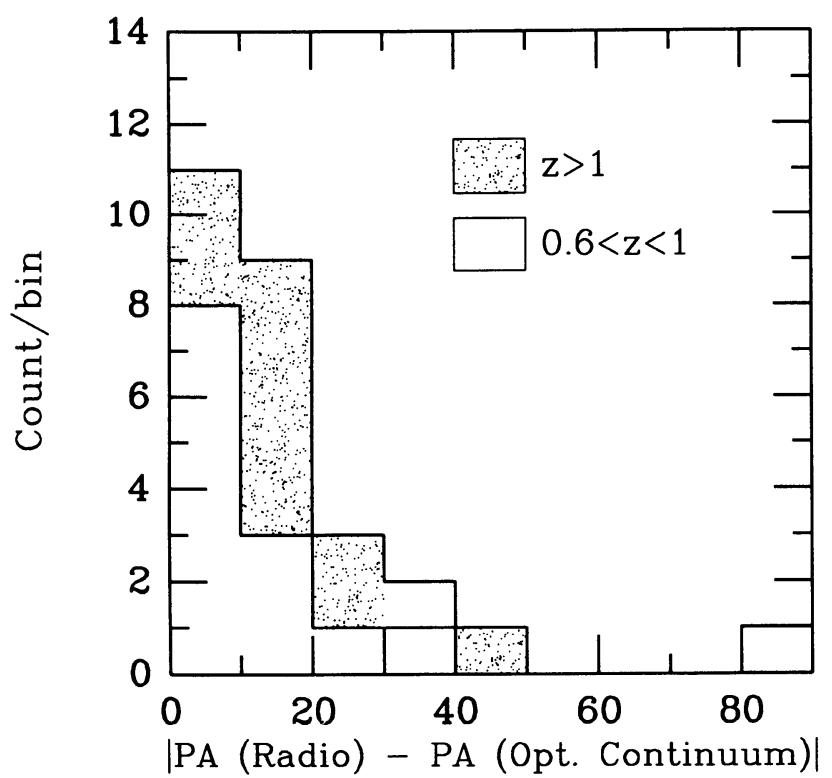

FIG. $3 a$

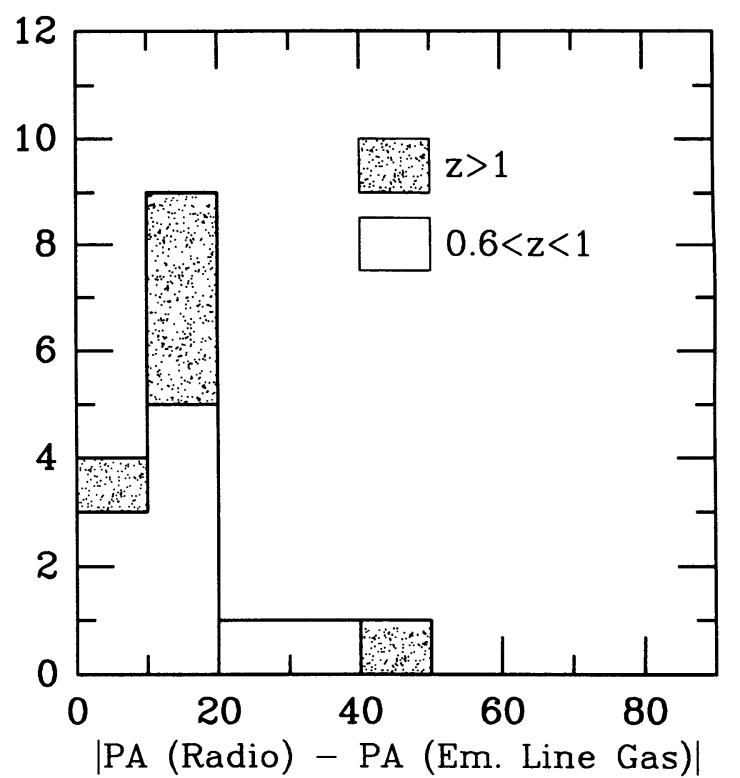

FIG. $3 b$

FIG. 3.- (a) Histograms of the differences between the radio and optical continuum position angle for distant $3 \mathrm{CR}$ galaxies. The position angle differences have been divided into $10^{\circ}$ bins. The shaded region is for galaxies with $z>1$, while the unshaded region is for galaxies with $1.0>z>0.6$. (b) Same as $(a)$ except the position angle of the extended emission-line gas is compared to that of the radio source. 
simple merger scenario the long axis of the system should not be preferred axis for the radio source, if any axis is favored at all. Clearly, gravity alone is incapable of producing the alignments that we observe.

This leads us to consider a scenario in which outflow along the radio source axis strongly influences the efficiency of star formation in this direction. In addition to the alignment of the optical continua with the radio axes, several other pieces of evidence, outlined below, suggest that interactions between these distant radio sources and their environments are important.

The large redshifts and blue colors of the sample galaxies ensure that most will have their $B, V$, and $R$ continuum images dominated by spectral types of $A$ and earlier. The short lifetime of these stars compared to the crossing time implies that we are seeing them in essentially the same location where they formed; hence there must be large star-forming complexes located along the radio source axes.

Nearly all of the 18 objects for which we have obtained narrow-band images showed extended extranuclear ionized gas. A preliminary comparison of these images with available radio data shows that this gas tends to lie along the radio source axes (Fig. $3 b$ ). While in most cases the line emission is located interior to the hot spots, with no obvious counterparts visible in the currently available radio data, in at least three cases (3C 280, 3C 337, and 3C 247) the [O II] emission is directly associated with one of the radio hotspots. One example (3C 280, $z=0.9978$ ) is shown in Figure 4 (Plate L5). The presence of weak continuum underlying the extended emission-line gas appears to be a general characteristic of distant $3 \mathrm{CR}$ radio galaxies and is suggestive of a source of in situ ionization, in contrast to the extended [O III] emission-line regions associated with nearby quasars (Stockton and MacKenty 1987). The properties of the extended emission-line regions in the distant $3 \mathrm{CR}$ galaxies are similar in some respects to the gas associated with nearby radio galaxies of lower luminosity (e.g. 3C 171, Heckman, van Breugel, and Miley 1984; 4C 29.30, van Bruegel et al. 1986).

Long-slit spectroscopic observations show that the majority of the objects in the sample with extended line emission exhibit large velocity gradients (up to $\sim 1500 \mathrm{~km} \mathrm{~s}^{-1}$ ). A long-slit spectrogram of 3C 280 is shown in Figure 5 (Plate L6). The extended [O II] emission seen in this object shows a large velocity gradient $\left(\sim 800 \mathrm{~km} \mathrm{~s}^{-1}\right.$ over $\left.\sim 50 \mathrm{kpc}\right)$ at the location of the eastern radio hot spot.

Numerical simulations (Norman et al. 1982) have shown that the terminal hot spots of radio jets have turbulent backflows that are efficient in entraining ambient material. We propose that the enhanced star formation along the radio axes of the distant radio galaxies may be due to entrainment and additional ram pressure exerted on the ambient gas by the backflows, as suggested for nearby low-luminosity radio galaxies by De Young (1981). Alternatively, the enhanced star formation might be induced by other outflow modes (e.g., nuclear star formation winds) accompanying the radio source phenomenon (e.g., van Breugel et al. 1986).

If the radio sources are to stimulate excess star formation, we must require that the age and size of the radio source be equal to or greater than those of the star formation regions. Simple estimates of the lifetimes of powerful doubles with sizes of $\sim 300 \mathrm{kpc}$ (the median size in our sample) are $\sim 10^{7}-10^{8}$ yr (e.g., Gopal-Krishna and Wiita 1987), and are thus comparable to or greater than the lifetime of the $\mathrm{O}$ and $\mathrm{B}$ stars that dominate our optical continuum images. Bursts of star formation triggered by the radio source could naturally explain the rapid evolution of the stellar populations of $3 \mathrm{CR}$ galaxies that has been deduced from photometric studies (e.g., Lilly and Longair 1984; Spinrad and Djorgovski 1987).

We recall that nearby radio galaxies are typically rounder than radio-quiet ellipticals, and in strong core sources the radio axis tends to be normal to the major axis of the stellar system. This may be due to the fact that in the distant galaxies we are comparing the distribution of a young stellar population to the radio source rather than the old populations that dominate nearby elliptical galaxies. It remains to be determined if the elongated morphologies that we observe at large redshifts are characteristic of the underlying mass distribution, implying that radio galaxies undergo strong dynamical evolution between $z \approx 1$ and the present, or if we are only sampling a young but dynamically insignificant fraction of the stellar population. Resolution of this question is important if we are to understand the formation and evolutionary histories of powerful radio galaxies and their relation to radio quiet giant ellipticals.

We would like to thank the staffs of the Kitt Peak National Observatory, the Canada-France-Hawaii telescope and the Lick Observatory for their expert assistance with the observations. We wish to express our thanks to M. Dickinson for help in CCD reductions and photometry, and to $S$. Kulkarni for the Palomar $5 \mathrm{~m}$ images of $3 \mathrm{C} \mathrm{437.} \mathrm{H.} \mathrm{S.} \mathrm{and} \mathrm{P.} \mathrm{McC.}$ acknowledge support from NSF grant AST85-13416. W. v. B. acknowledges support from NSF grant AST84-16177, and S. D. acknowledges partial support from Harvard University.

\section{REFERENCES}

Baum, S. 1986, Bull. $A A S, 18,1004$

Bridle, A. H., and Brandie, G. W. 1973, Ap. Letters, 15, 21.

Brodie, J. P., Bowyer C. S., and McCarthy P. J. 1985, Ap. J. (Letters), 293, L25.

Chambers, K. C., Miley, G. K., and van Breugel, W. J. M. 1987, Nature, in press.

Cordey, R. A. 1986, M.N.R.A.S., 219, 575.

De Young, D. S. 1981, Nature, 293, 43.

Djorgovski, S. 1987, in Nearly Normal Galaxies, ed. S. Faber (Berlin: Springer), p. 290.

Djorgovski, S., Spinrad, H., Pedelty, J., Rudnick, L., and Stockton, A. 1987, A.J., 93, 1307.

Frenk, C., White, S. D. M., Davis, M., and Efstathiou, G. 1987, Ap. J., submitted.
Graham, J. A., and Price, R. M. 1981, Ap. J., 247, 813.

Gopal-Krishna, X., and Wiita, P. 1987, preprint.

Heckman, T. M., Smith, E. P., van Breugel, W. J. M., Balick, B., Miley, G. K., Bothun, G. D., Illingworth, G. D., and Baum, S. A. 1986, Ap. J., 311, 526.

Heckman, T. M., van Breugel, W. J. M., and Miley, G. K. 1984, Ap. J., 286, 509.

Hutchings, J. B., 1987, preprint.

Jenkins, C. J., Pooley, G. G., and Riley, J. M. 1977, Mem. R.A.S., 84, 61.

Kapahi, V. K., and Saikia, D. J. 1982, J. Astr. Ap., 3, 161

Le Fevre, O., Hammer, F., Nottale, L., and Mathez, G. 1987, Nature, 326, 268.

Lilly, S., and Longair, M. 1984, M.N.R.A.S., 211, 833. 


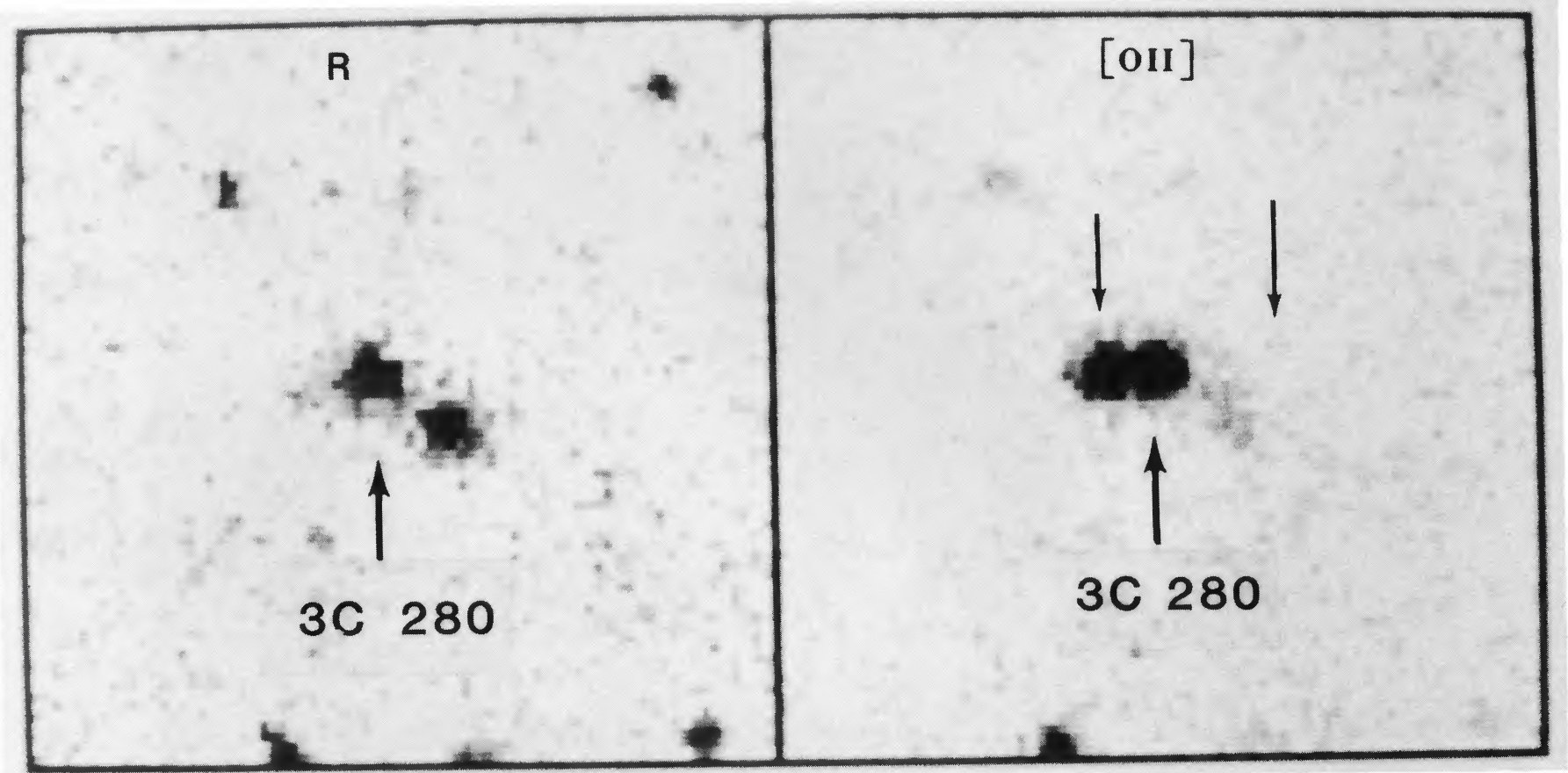

FIG. 4. - A composite of a broad-band $r$ image (left) and an [O $\mathrm{II}] \lambda 3727$ image (right) of the radio galaxy $3 \mathrm{C} 280$ ( $z=0.997)$. The locations of the radio lobes are indicated with the upper arrows; the location of the host galaxy is marked by the lower arrow. The size of each panel is $55^{\prime \prime} \times 55^{\prime \prime}$. Note that the extended [ $\mathrm{O}$ II] emission is spatially coincident with the eastern radio hot spot and the $r$ image shows a faint continuum source at this location.

MCCARTHY et al. (see 321, L32) 


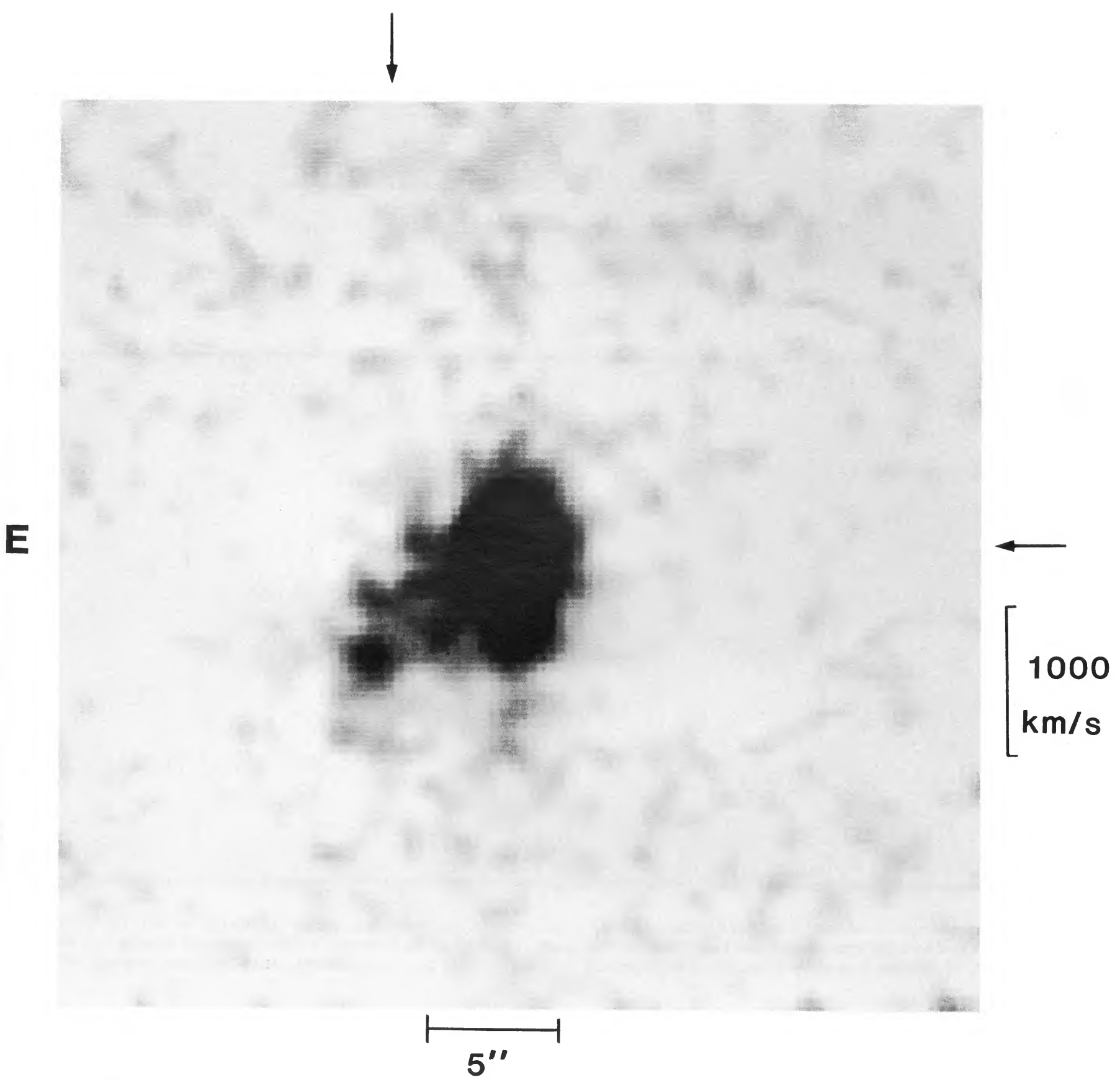

FIG. 5.-A section of a long slit spectrogram of the extended emission-line gas associated with 3C $280(z=0.997)$. The spectrogram was taken with the slit aligned along the radio source axis $\left(\mathrm{PA}=91^{\circ}\right)$. The $X$-axis and $Y$-axis are labeled with distance in arcseconds and velocity in km $\mathrm{s}^{-1}$, respectively. The systemic velocity and the position of the eastern radio hot spot are marked with the horizontal and vertical arrows, respectively. The spectrogram was taken with the Lick Shane $3 \mathrm{~m} \mathrm{CCD}$ spectrograph and has $9 \AA$ resolution.

MCCARTHY et al. (see 321, L32) 
Mackay, C. D. 1971, M.N.R.A.S., 151, 421.

McCarthy, P. J., Spinrad, H., Djorgovski, S., Strauss, M. A., van Breugel, W. J. M., and Liebert, J. 1987, Ap. J. (Letters), 319, L39.

Norman, M. L., Smarr, L., Winkler, K.-H., and Smith, M. D. 1982, Astr. Ap., 113, 285.

Spinrad, H. 1986, Pub. A.S.P., 98, 269.

in 1987, in KPNO Conference on Large Telescopes and Cosmology,

Spinrad, H., and Djorgovski, S. $1984 a$, Ap. J. (Letters), 280, L9. $1984 b$, Ap. J. (Letters), 285, L49. . 1984 c, Pub. A.S.P., 96, 795.
Spinrad, H., and Djorgovski, S. 1987, in IAU Symposium 124, Observational Cosmology, ed. G. Burbidge (Dordrecht: Reidel), p. 129.

Spinrad, H., Djorgovski, S., Marr, J., and Aguilar, L. A. 1985, Pub. A.S.P., 97, 932 .

Stockton, A., and MacKenty, J. 1987, Ap. J, 316, 584

van Breugel, W. J. M. 1986, Canadian J. Phys., 64, 392.

van Breugel, W. J. M., Filippenko, A. V., Heckman, T. M., and Miley, G. K. 1985, Ap. J., 293, 83.

van Breugel, W. J. M., Heckman, T. M., Miley, G. K., and Filippenko, A. V. 1986, Ap. J., 311, 58.

S. DJoRgovski: Palomar Observatory, 105-24 CalTech, Pasadena, CA 91125

Patrick J. McCarthy and HYron SpINRad: Department of Astronomy, University of California, Berkeley CA 94720

WIL J. M. vAN BREUGeL: Radio Astronomy Laboratory, University of California, Berkeley CA 94720 